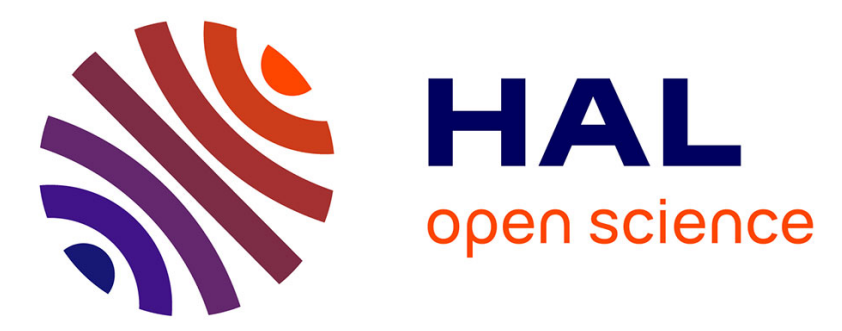

\title{
Measuring yarn deformations induced by the manufacturing process of woven composites
}

Arturo Mendoza, Julien Schneider, Estelle Parra, Stéphane Roux

\section{To cite this version:}

Arturo Mendoza, Julien Schneider, Estelle Parra, Stéphane Roux. Measuring yarn deformations induced by the manufacturing process of woven composites. Composites Part A: Applied Science and Manufacturing, 2019, 120, pp.127-139. 10.1016/j.compositesa.2019.02.008 . hal-02156837

\section{HAL Id: hal-02156837 https://hal.science/hal-02156837}

Submitted on 14 Jun 2019

HAL is a multi-disciplinary open access archive for the deposit and dissemination of scientific research documents, whether they are published or not. The documents may come from teaching and research institutions in France or abroad, or from public or private research centers.
L'archive ouverte pluridisciplinaire HAL, est destinée au dépôt et à la diffusion de documents scientifiques de niveau recherche, publiés ou non, émanant des établissements d'enseignement et de recherche français ou étrangers, des laboratoires publics ou privés. 


\title{
Measuring yarn deformations induced by the manufacturing process of woven composites
}

\author{
Arturo Mendoza ${ }^{\mathrm{a}, \mathrm{b}}$, Julien Schneider ${ }^{\mathrm{c}}$, Estelle Parra ${ }^{\mathrm{b}}$, Stéphane Roux ${ }^{\mathrm{a}}$ \\ ${ }^{a}$ LMT (ENS Paris-Saclay/CNRS/Univ. Paris-Saclay), \\ 61 Avenue du Président Wilson, 94235 Cachan, France \\ ${ }^{b}$ Safran Tech, Rue des Jeunes Bois, 78772 Magny les Hameaux, France \\ ${ }^{c}$ Safran Aircraft Engines, Rond Point Réné Ravaud - Réau, 77550 Moissy-Cramayel, France
}

\begin{abstract}
A new technique is presented for measuring the yarn deformations induced by the complete manufacturing process of woven composites. This approach relies on a well suited relative analysis using Digital Volume Correlation [1]. Two pairs of composite samples observed through high-resolution X-ray computed tomography are used as illustration. The measured differences between samples allows identifying a clear typology of strain patterns. They are in good agreement with phenomena known by experts but whose analysis is traditionally performed through manual inspection of tomographic data. For such reasons, these results are exploited using machine learning techniques so as to automate the unsupervised identification of these strain patterns. These results show the potential of the method in terms of automated inspection of 3D woven textiles for quality control, or for fine adjustment of manufacturing parameters.
\end{abstract}

Keywords: A. Fabrics/textiles, C. Numerical analysis, D. CT analysis, E. Resin transfer moulding (RTM)

\section{Introduction}

The manufacturing of 3D woven composites typically consist of (i) the weaving of the yarns according to the weaving pattern, (ii) the subsequent preforming

\footnotetext{
${ }^{*}$ Corresponding author

Email address: mendoza@lmt.ens-cachan.fr (Arturo Mendoza)
}

Preprint submitted to Composites Part A: Applied Science and ManufacturingJune 14, 2019 
of the obtained textile reinforcement (iii) and the matrix infusion using resin transfer molding (RTM). During each of these steps the yarns are subjected to large local and global deformations, which lead to a non-negligible variability in the internal geometry of textile composites. These differences, observed in reference [2], were revealed via high resolution X-ray computed tomography (micro-CT). Since this variability affects many specific (macro-)properties of the material, much well deserved attention has been given to the deformations that the yarns undergo (meso-scale) during the manufacturing process [3].

First, a better understanding of the forming process has been achieved. Experimental data were essential for validating the numerical simulation models [3, 4]. Digital Image Correlation (DIC) [5-7] and stereo-DIC (or 3D-DIC) [8] have provided valuable data for the surface characterization of the forming process of (thin) specimens, and consequently have enabled the validation of many numerical models [9-16].

DIC and stereo-DIC are well established techniques for accurately measuring the $2 \mathrm{D}$ displacements fields relating image pairs. While DIC requires only one point of view, stereo-DIC requires multiple (at least two) views so as to provide the 3D surface displacement. Both techniques benefit from long series of images acquired during the deformation of the sample. These experimental techniques are popular due to their relatively simple setup and rich full-field results.

Likewise, Digital Volume Correlation (DVC) [17, 18] is the true threedimensional extension to DIC. DVC allows the measurement of internal displacement fields from volume pairs (3D images), such as those obtained from X-ray computed tomography $(\mathrm{CT})$.

At this point, it is important to note that current works on the forming process rely on the observed (and measured) deformations at the surface of the samples. Naturally, a deeper insight into the composite processes calls for an analysis of the entire sample (i.e., micro-CT) using a full 3D measurement tool (i.e., DVC). However, while it is possible to acquire many surface images of the preform during its deformation, it is not feasible to do the same for tomography. Hence the amount of experimental 3D data would be extremely reduced (e.g., 
only before and after forming) and may not be enough for accurately performing traditional DVC.

Second, it has recently become clear that the correct determination of the textile configuration (due to the weaving process) is paramount for the study of the forming process [19]. Therefore, models based on the "nominal" (theoretical) geometry of the textile may be undesirable, in fact, current models are based on a more realistic "as-woven" geometry of the reinforcement [20]. These geometries can be obtained through simulations of the textile process, in which yarns are modeled either as "solid" (or "tubular") entities [21-26] or as "multi-filament" ("multi-chain") entities [19, 27-33]. These models can be initialized via some ad hoc techniques (e.g., pre-tensioning using a fictitious thermal expansion) or just by accessing the weaving architecture [33]. Alternatively, this configuration can be directly extracted from high resolution CT (micro-CT) [34, 35], so as to account for the specificities of the analyzed material.

In such sense, the former approach could be seen as the most advantageous one. In fact, geometry modeling allows obtaining textile models without actually manufacturing the specimen [26]; thus allowing a multitude of analyses to be rapidly performed. On the contrary, CT volume data can be (considerably) big and require heavy manual operations in order to identify the elements of interest. Besides, the available tools for performing these operations are either not generic enough or they rely heavily on the expertise of the operator e.g., manual inspection. As a result, CT analyses can be regarded as an approach requiring considerable amounts of tedious work that only amount to information for one sample (the one being studied). Moreover, each sample to be analyzed demands the same amount of investment.

Despite these issues, the analysis of micro-CT (3D images) for the measurement of yarn deformations during the whole manufacturing process could become an attractive and streamlined alternative thanks to recent developments in DVC $[1,36]$ for the comparative analysis of 3D woven composites. The method is based on an assumption of constant topology, namely, the specific ordering or crossing of the different yarns, is preserved; hence, it aligns perfectly 
with the current goal. Multiple specimens can be analyzed so as to obtain the relative yarn displacement and deformations (i.e., the so-called "metric differences"). Furthermore, a newly developed "complete" mechanical regularization technique [36] (based on the equilibrium gap [37-39]) should prove helpful.

One of the many advantages of this approach is its striking simplicity. Indeed, it does not require any custom or complex priors about the material, the textiles themselves (as seen through $\mathrm{CT}$ ) provide all the necessary information in tremendous amounts. Hence, it allows identifying complex phenomena without any particular effort in describing them previously nor in seeking them actively. This results in a (fast) analysis capable of shedding light into phenomena that may be unknown (or unexpected) at the time of the analysis.

Additionally, the measured information can help gain a better understanding of some textile aspects. If desired, this knowledge could be interpreted with respect to any of the many factors involved in the manufacturing process. Such factors include the textile definition (e.g., weaving pattern) [40], the handling of the preform, the draping strategy, amongst others. In sum, the advantages provided by this strategy should overcome the "inconveniences" evoked earlier with regards to the use of micro-CT data.

The details about the correlation procedure are detailed in Section 2. The available data set and preparation for analysis is presented in Section 3, they consist of two pairs of 3D woven composite samples. The results from these analyses are shown in Section 4, they detail the results for each pair independently as well as the advantages of "combining" both results. These show the advantages of the proposed method for the analysis of yarn deformations in woven composites due to the complete manufacturing process.

\section{Method}

\subsection{Correlation procedure}

The goal of the correlation procedure is the registration of a given pair of images $f(\boldsymbol{x})$ and $g(\boldsymbol{x})$. These images correspond to the reference and test (i.e., 
deformed) configurations respectively. Then, the objective translates to finding the displacement field $\boldsymbol{u}(\boldsymbol{x})$ that minimizes the brightness conservation residuals $\eta(\boldsymbol{x})$

$$
\eta(\boldsymbol{x})=g(\boldsymbol{x}+\boldsymbol{u}(\boldsymbol{x}))-f(\boldsymbol{x})
$$

where $\eta(\boldsymbol{x})$ captures all the phenomena not considered in the registration. In the present case (X-ray micro-CT), they ideally correspond to only noise; but, there are also some instances of tomographic reconstruction artefacts (e.g., ring artefact).

As such, the sought displacement is the one that minimizes, over the region of interest $\Omega$, the $L_{2}$ norm of the residuals $\eta(\boldsymbol{x})$

$$
\phi=\int_{\Omega} \eta(\boldsymbol{x})^{2} \mathrm{~d} x
$$

This optimization problem is an ill-posed one. Hence, $\boldsymbol{u}(\boldsymbol{x})$ is constraint to a space of lower dimension by decomposing the displacement field over a set of known base functions $\psi$, such as those from the Finite Element (FE) method [41]

$$
\boldsymbol{u}(\boldsymbol{x})=\sum_{i} u_{i} \boldsymbol{\psi}_{i}(\boldsymbol{x})
$$

Thus obtaining a "global" [42] approach that guarantees the continuity of the solution, as well as the inter-dependence of the sought amplitudes $u_{i}$.

Finally, an iterative Newton-Raphson scheme [43] leads to the following linear system

$$
[\boldsymbol{M}]\{\delta \boldsymbol{u}\}=\{\boldsymbol{b}\}
$$

where the matrix $[\boldsymbol{M}]$ embeds the image gradients $\boldsymbol{\nabla} f(\boldsymbol{x})$ and the shape functions $\boldsymbol{\psi}_{i}(\boldsymbol{x})$, the vector $\{\boldsymbol{b}\}$ accounts for the image residuals $\eta(\boldsymbol{x})$, and $\{\delta \boldsymbol{u}\}$ updates the degrees of freedom $\{\boldsymbol{u}\}$ after each iteration.

\subsection{Correlation for textiles}

While "classical" DVC deals with the evolution of one sample, the intended use case deals with different composites samples [1].

As previously introduced, the approach is based on the assumption of constant topology and provides a robust formulation of the correlation problem. 
This novel formulation considers a relaxed brightness conservation assumption so as to allow for gray level variations that result from phenomena not explained by the assumption of topology (e.g., weaving anomalies, tomographic artefacts).

Furthermore, regularization techniques are employed so as to better condition the problem. First, the displacement field is regularized using a "complete" mechanical regularization scheme [36]. This favors displacement field locally obeying a prescribed behavior (e.g., linear elasticity). Second, the gray level correction fields are regularized by the $L_{2}$ norm of their Laplacian. Both strategies can be seen as a set of filters that locally dampen steep gradients and ensure smooth and differentiable fields.

Finally, the correlation is performed under a multiresolution strategy that consists in considering at first coarse images to evaluate the largest displacements, and progressively images with a finer and finer resolution pre-corrected by the previously estimated displacement fields to evaluate the details of the dis-

placement field. Hence, big textile displacements and small yarn deformations can be both captured by the method.

\section{Setup}

Four composite samples sharing the same weaving pattern (i.e., relative yarn positions) are considered in the present analysis: S1, S2, S3 and S4. In order to ease the understanding of the results, two pairs of samples are proposed: S1-S2 and S3-S4.

The samples are observed using X-ray micro-CT. The available observed region for pair S1-S2 contains an entire unit cell, and the observation of pair S3-S4 is done over a wider region. Since the target scale is the meso-scale, the voxel size is coarse enough so as to "average" the information at the micro-scale (i.e., the carbon fibers), while being sufficiently fine so as to allow a proper description of the textile (i.e., the yarns). Similarly, low energy X-rays are employed in order to obtain the maximum contrast between phases. As such, the reconstructed volumes differentiate the (carbon) yarns and the (polymer) 
resin.

All studied samples are oriented so that the $x$ axis corresponds to the weft orientation, the $z$ axis to the warp orientation, and the $y$ axis to the transversal orientation. Some 2D slices for both pairs of samples, shown in figures 1 and 2, illustrate on the variability of the considered samples.

Finally, the "correlation for textiles" is performed on both pairs. The samples S1 and S3 are chosen as references for their respective pairs; therefore, samples S2 and S4 are considered in a "deformed" configuration. The kinematic description for the pair S1-S2 (resp. S3-S4) is decomposed over 9600 (resp. 22572) degrees of freedom using a regular structured mesh.

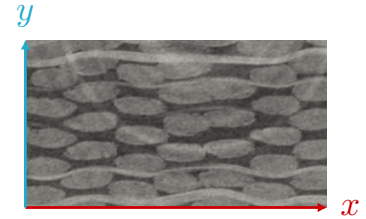

(a) S1

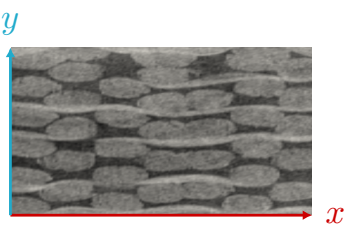

(c) S2

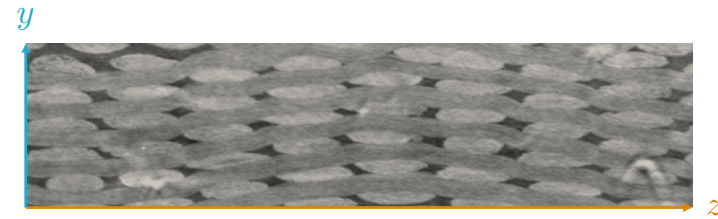

(b) S1

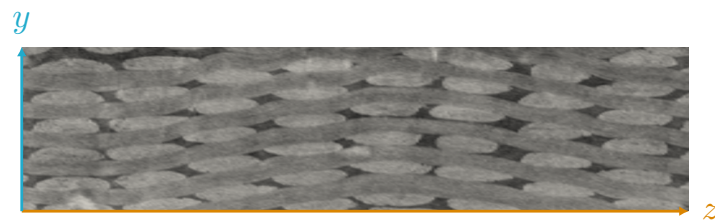

(d) S2

Figure 1: $(x-y)$ and $(y-z)$ slices for pair S1-S2, they correspond to "equivalent" regions in the composite 


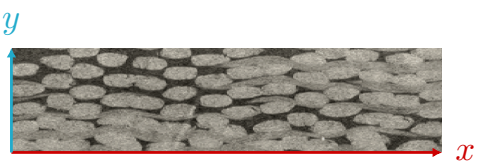

(a) S3

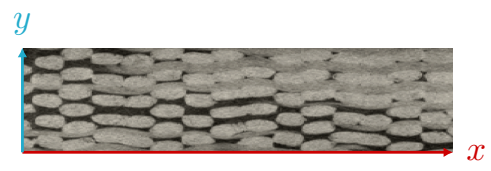

(c) S4 y

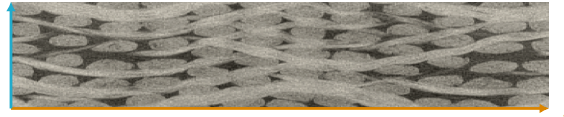

(b) S3

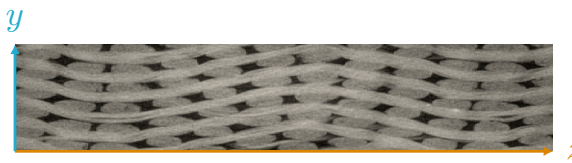

(d) S4

Figure 2: $(x-y)$ and $(y-z)$ slices for pair S3-S4, they correspond to "equivalent" regions in the composite

\section{Results}

The obtained results for each pair (S1-S2 and S3-S4) will be explored in the following. First, the analysis of each pair will be performed independently so as to gain insights into the different phenomena induced by the entire manufacturing process. Then, the strain patterns identified in the second pair will be used for describing those of the first pair. This step will demonstrate the advantages of using a common "language" in the analysis of woven composite deformations.

Given the nature of the found displacement fields, it is proposed to carry out their analysis via their relative strains [44], presented in the form of the strain tensor $\boldsymbol{\epsilon}(\boldsymbol{x})$ field. They inform on the deformations that the test samples (S2 and S4) need to undergo to resemble their respective references (resp. S1 and S3). Thus the relative nature of the descriptor. The logarithmic (or Hencky) strain [45] definition is chosen to account for large strains as well as for large rotation [46], while excluding rigid-body motion of the medium.

It should be noted that this second-order strain tensor is symmetric (i.e., 


$$
\left.\epsilon_{i j}=\epsilon_{j i}\right)
$$

and can be represented as a vector using the Voigt notation (order reduction) [47]

$$
\boldsymbol{\epsilon}=\left(\epsilon_{x x}, \epsilon_{y y}, \epsilon_{z z}, \epsilon_{y z}, \epsilon_{x z}, \epsilon_{x y}\right)
$$

Additionally, two invariant quantities (with respect to any coordinate system) can be obtained from the strain tensor: the $\operatorname{trace} \operatorname{tr}(\boldsymbol{\epsilon})$ and the equivalent strain $\boldsymbol{\epsilon}_{e q}[48,49]$

$$
\begin{aligned}
\operatorname{tr}(\boldsymbol{\epsilon}) & =\epsilon_{x x}+\epsilon_{y y}+\epsilon_{z z} \\
\boldsymbol{\epsilon}_{e q} & =\sqrt{\frac{2}{3}} \cdot \sqrt{\operatorname{tr}\left(\boldsymbol{\epsilon}^{2}\right)-\frac{1}{3} \operatorname{tr}(\boldsymbol{\epsilon})^{2}}
\end{aligned}
$$

The former accounts for normal deformations (volume change), while the latter considers shear deformation magnitude (at constant volume).

Finally, principal strains are a useful tool for analyzing the strain tensor. They are the eigenvalues of the strain tensor, and are labeled such that $\epsilon_{1} \geq$ $\epsilon_{2} \geq \epsilon_{3}$

The formalism introduced by the principal strain tensor allows a tensor glyph visualization using ellipsoids $[50,51]$. Their axes are determined by the eigenvectors (principal orientations) and their diameters are determined by the eigenvalues (components of the strain tensor). This visualization is useful for identifying, at a glance, different types of strain patterns; for example, when the ellipse approximates a sphere the strain state is isotropic. Similarly, a stretch along a direction and or in a plane are represented by the ellipse approximating a "cigar" or "pancake" shape, respectively.

Finally, all displacement and strain measurements presented herein will be normalized with respect to a pair of chosen key values $u_{o}$ and $\epsilon_{o}$. 


\subsection{Analysis of pair S1-S2}

The registration of this first pair can be verified through the image of residuals $\eta(\boldsymbol{x})$ and the displacement field $\boldsymbol{u}(\boldsymbol{x})$.

The former informs on the quality of the registration: the residual $\eta(\boldsymbol{x})$ is centered at zero with a standard deviation of $7.8 \%$ of the initial 16 bits dynamic range. Additionally, all the remaining high-valued residuals are easily identified as ring artifacts (i.e., tomographic reconstruction), as seen in figure 3a. Such behavior is highly desirable in view of the fact that even "imperfect" tomographic images do not alter the DVC analysis, thus its result is deemed reliable.

The displacement field is shown in figures $3 \mathrm{~b}$ to $3 \mathrm{~d}$ for each of its components. A noteworthy observation is that $u_{y} \approx \mathbf{0}$, so $\boldsymbol{u}$ is essentially parallel to the $(x-z)$ plane and varies mostly with $y$. This layered kinematics with all $(x-z)$ planes being mostly translated along their plane, can also be observed from $u_{x}$ and $u_{z}$. It makes sense because such "sliding" between the layers of the woven composite is to be expected. In fact, each layer is essentially a $2 \mathrm{D}$ textile which cannot be stretched neither along the weft nor the warp orientation (i.e., $\epsilon_{x x}=\epsilon_{z z}=0$ ). This leaves mostly rigid translation in the $(x-z)$ plane with minimal shear $\epsilon_{x z}$; hence, the most significant strains are to be expected as shear in the planes $(x-y)$ and $(y-z)$. A volume representation (iso-curves) of the strains $\epsilon_{x y}$ and $\epsilon_{y z}$ is shown in figures $4 \mathrm{a}$ and $4 \mathrm{~b}$.

Furthermore, it is possible to visualize the deformations with respect to the composite samples, as shown in figures $4 \mathrm{c}$ and $4 \mathrm{~d}$ for the different views of sample $\mathrm{S} 1$ (reference), and in figures $4 \mathrm{e}$ and $4 \mathrm{f}$ for the sample $\mathrm{S} 2$. As the figures $4 \mathrm{c}$ to $4 \mathrm{f}$ show, the identified phenomena are strongly related to the meso-structure of the composite. Furthermore, some highly localized strain patterns can be identified: The first strain pattern B1, observed between figure 4c and figure 4e, is the same "sliding" of layers described earlier. The second strain pattern B2, observed between figure $4 \mathrm{~d}$ and figure $4 \mathrm{f}$, shows a similar "sliding" of yarns at an oblique orientation. Here, the bundle of yarns to left (resp. right) of the oblique plane slide left-downwards (resp. right-upwards). It should be noted that these qualitative description of the strain patterns B1 and B2 is motivated 
by the underlying quantitative measurements.

Finally, on account of the predominant shear strain, the equivalent strain is chosen for analysis, multiple $(x-y)$ and $(y-z)$ views for the $\epsilon_{e q}$ and $\mathrm{S} 1$ are shown in figure 5. It can be seen that $\epsilon_{e q}$ is highly localized in a region constrained between two weft layers and comprises three warp columns. The layered kinematics described earlier is mostly expressed in this region. It is also noteworthy that the results are highly localized despite the considerable ring artefact.

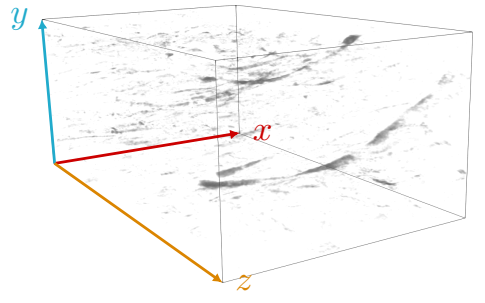

(a) $\eta(\boldsymbol{x})$

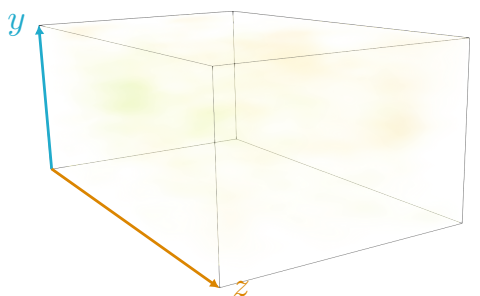

(c) $u_{y}(\boldsymbol{x})$

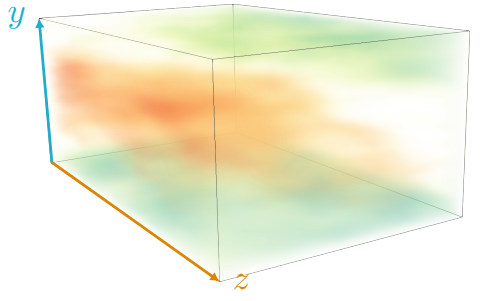

(b) $u_{x}(\boldsymbol{x})$

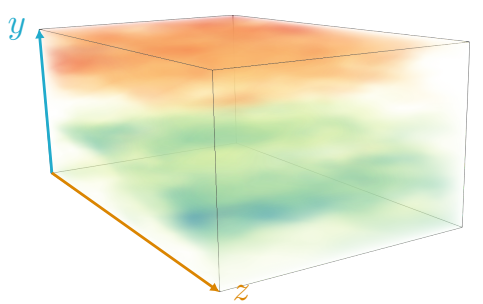

(d) $u_{z}(\boldsymbol{x})$

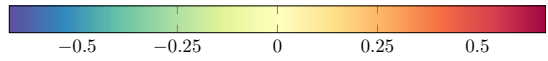

Figure 3: Results of correlation for pair S1-S2: (a) image of residuals and (b)-(d) components of the displacement field (normalized with respect to $u_{o}$ ) 


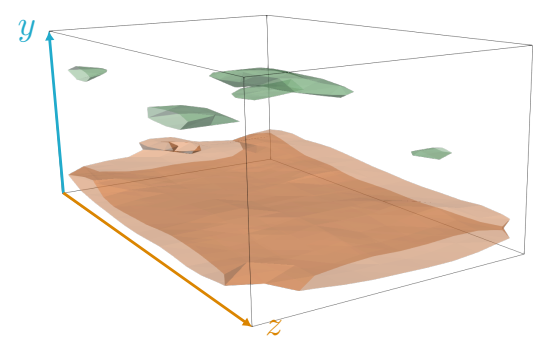

(a) $\epsilon_{x y}$

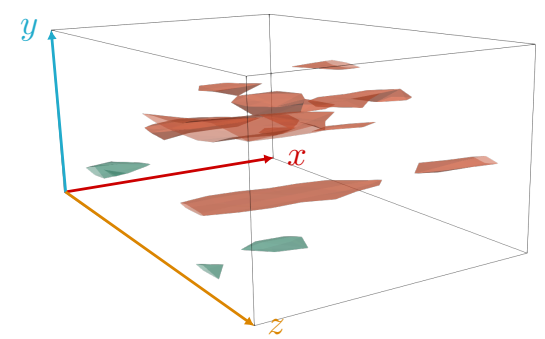

(b) $\epsilon_{y z}$

$y$

y

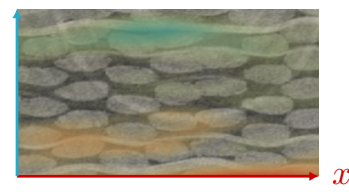

(c) $\epsilon_{x y}$ on $\mathrm{S} 1$

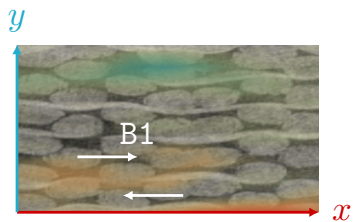

(e) $\epsilon_{x y}$ on $\mathrm{S} 2$

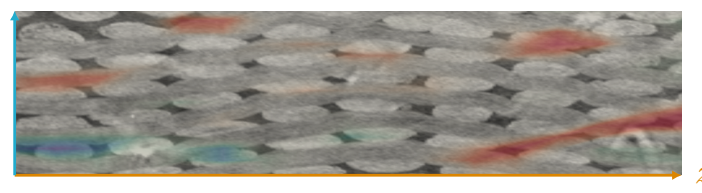

(d) $\epsilon_{y z}$ on $\mathbf{S} 1$

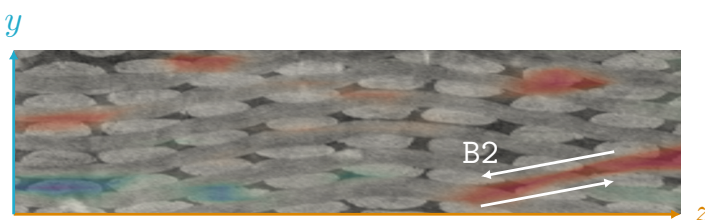

(f) $\epsilon_{y z}$ on $\mathrm{S} 2$

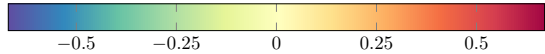

Figure 4: (a)-(b) Selected components of the strain tensor $\boldsymbol{\epsilon}$ (normalized with respect to $\epsilon_{o}$ ) shown over the corresponding slices of samples (c)-(d) S1 and (e)-(f) $\mathrm{S} 2$ 


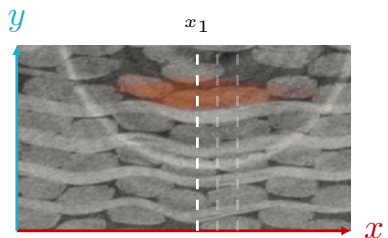

(a) $(x-y)$ view for $z_{1}$

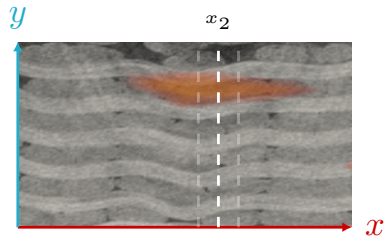

(c) $(x-y)$ view for $z_{2}$

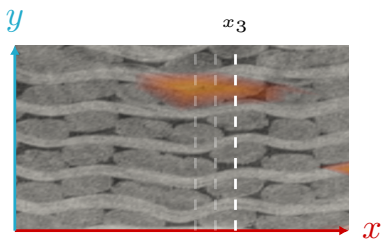

(e) $(x-y)$ view for $z_{3}$

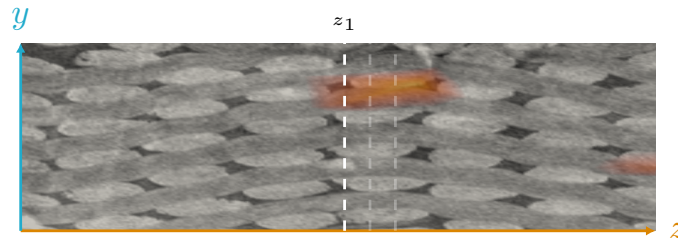

(b) $(y-z)$ view for $x_{1}$

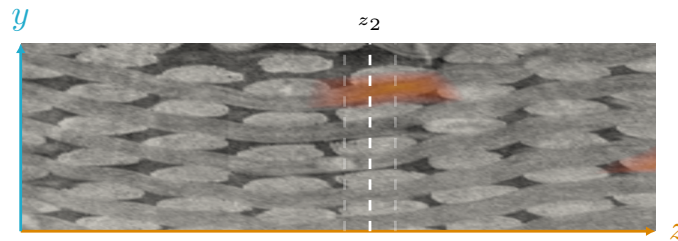

(d) $(y-z)$ view for $x_{2}$

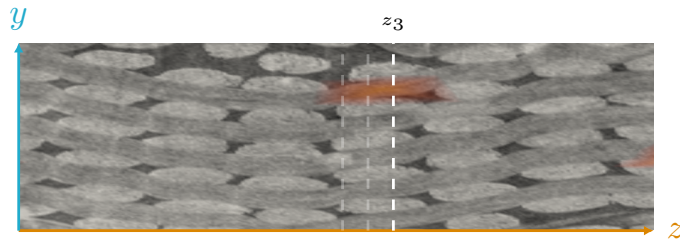

(f) $(y-z)$ view for $x_{3}$

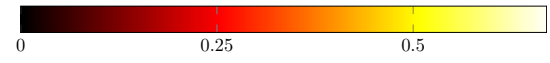

Figure 5: $(x-y)$ and $(y-z)$ views at different $z$ and $x$ positions of $\mathrm{S} 1$ and $\epsilon_{e q}$ (normalized with respect to $\epsilon_{o}$ ) 


\subsection{Analysis of pair $\mathrm{S3-S4}$}

The analysis of the registration between this second pair will purposely be different than previously. In fact, the goal of this section is to provide a new method of analysis for this type of results.

First, it can be helpful to analyze the displacement and deformation through some visualizations. The displacement field $\boldsymbol{u}(\boldsymbol{x})$, shown in figure $6 \mathrm{a}$, is applied over the reference support (mesh) and warped accordingly. This allows observing a slight shearing along with a depression on the $(y-z)$ surface that corresponds to $x=0$. Also, the strain tensor $\epsilon$ is shown in figure $6 \mathrm{~b}$ using a tensor glyph visualization.

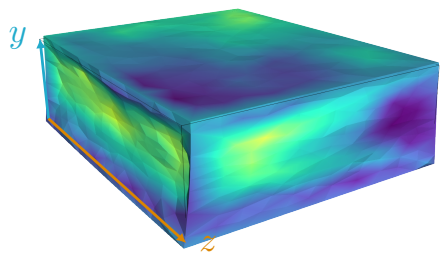

(a) $\|\boldsymbol{u}(\boldsymbol{x})\|_{2}$

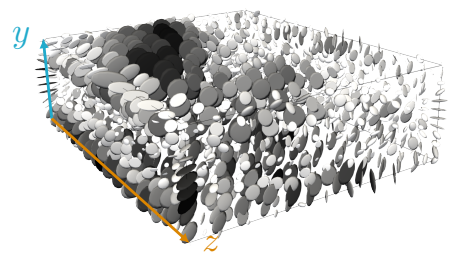

(b) $\epsilon$
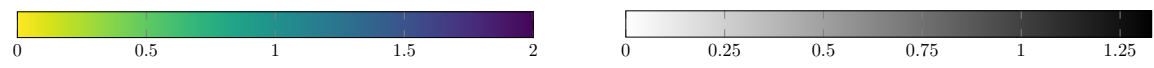

Figure 6: Results of correlation for pair S3-S4: (a) displacement field (normalized with respect to $u_{o}$ ) and (b) strain tensor (normalized with respect to the largest eigenvalue and $\epsilon_{o}$ )

Then, two components of the strain tensor are chosen for visualization: $\epsilon_{x x}$ and $\epsilon_{x y}$; These strains correspond to compression (or dilation) along the $x$ axis and shear in the $(x-y)$ plane, respectively; a volume representation (iso-curves) of the selected strains is shown in figures $7 \mathrm{a}$ and $7 \mathrm{~b}$. The impact of these fields on the composite samples is shown in figures $7 \mathrm{c}$ and $7 \mathrm{~d}$ for the sample S3 (reference), and in figures $7 \mathrm{e}$ and $7 \mathrm{f}$ for the sample $\mathrm{S} 4$. Again, it can be seen that the measured deformations are strongly related to the structure of the woven composite. For example, the "dislocation" pattern B3, seen in figures 7d 
and 7f, can be explained by the top- and bottom-most layers moving relative to the inner layers. However, a portion of the top-most layer (on the right side) "holds" onto its the neighboring layers. This results in the "sliding" being translated to an inner layer below, which is displayed as high values of shear in the $(x-y)$ plane; in turn, this creates in a region of high compression along the $x$ direction, as shown in figure $7 \mathrm{e}$.

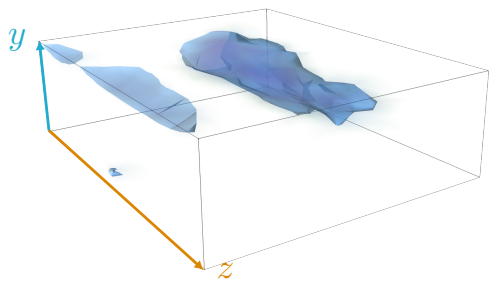

(a) $\epsilon_{x x}$

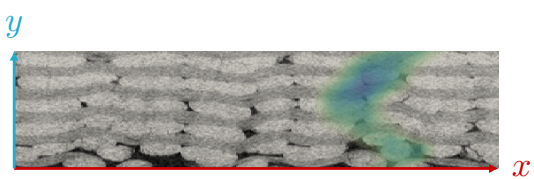

(c) $\epsilon_{x x}$ on $\mathrm{S} 3$

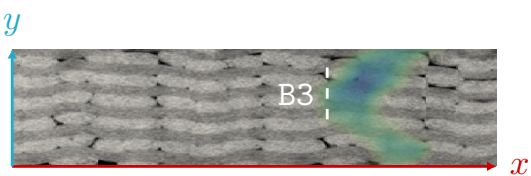

(e) $\epsilon_{x x}$ on S4

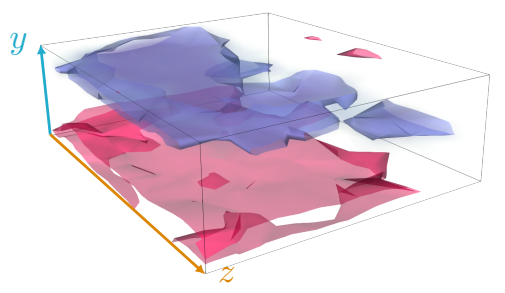

(b) $\epsilon_{x y}$

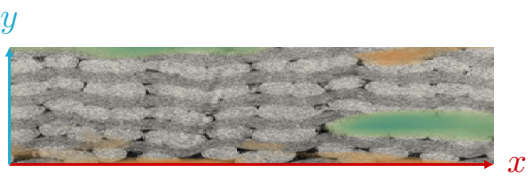

(d) $\epsilon_{x y}$ on $\mathrm{S} 3$

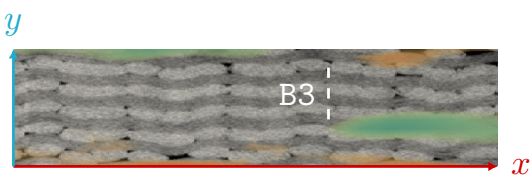

(f) $\epsilon_{x y}$ on $\mathrm{S} 4$

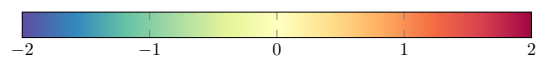

Figure 7: (a)-(b) Selected components of the strain tensor $\boldsymbol{\epsilon}$ (normalized with respect to $\epsilon_{o}$ ) shown over the corresponding slices of samples (c)-(d) S3 and (e)(f) S4 (different $z$ positions are shown in order to better illustrate the effects)

These previously described phenomena can be summarized in the diagram shown in figure 8. A region of high compression $\left(\epsilon_{x x}<<0\right)$ is created when most of the columns at inner layers (with $u_{x}>0$ ) "collide" with the remaining 
columns (with $u_{x}<0$ ). At the boundaries of these bundles of yarns some regions with high shear (with $\left|\epsilon_{x y}\right|>>0$ ) are created. Again, it is important to note that this qualitative description of the strain pattern B3 is motivated by the measured differences between the composite samples.

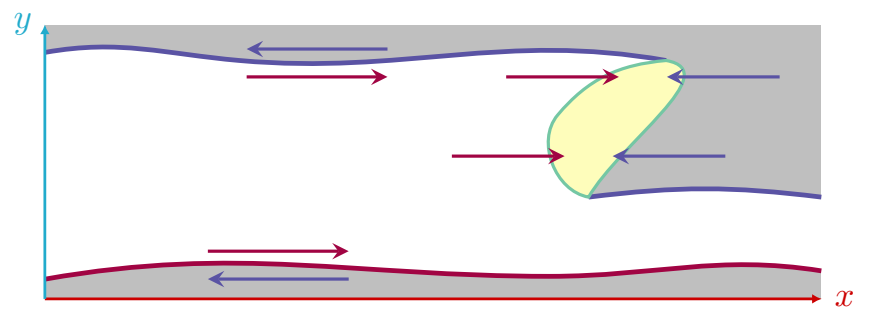

Figure 8: Diagram of observed phenomena for the pair S3-S4: $\square$ a compression zone is created (with $\epsilon_{x x}<<0$ ) when $\square$ a block moves right $\left(u_{x}>0\right.$ ) and the other blocks move left $\left(u_{x}<0\right)$; these are at the origin of shear in the $(x-y)$ plane, $\boldsymbol{\square}$ positive $\left(\epsilon_{x y}>>0\right)$ and $\boldsymbol{\square}$ negative $\left(\epsilon_{x y}<<0\right)$

\subsection{Distillation of results using machine learning}

This next part of the analysis consists in providing a set of quantitative descriptors for the measured differences that will be obtained by combining the known (observed) phenomena with unsupervised machine learning techniques. The reader not specifically interested in the technical details of this section can skip to section 4.4 .

The relative strain measurements are studied using the Principal Component Analysis (PCA) [52-54]. This technique consists on an orthogonal transformation that converts a dataset of possibly correlated variables into a set of linearly independent variables. Such transformation is obtained from the covariance matrix of the dataset

$$
\Sigma=X^{\top} \boldsymbol{X}
$$

and its eigendecomposition

$$
\boldsymbol{\Sigma}=\boldsymbol{Q} \Lambda Q^{-1}
$$


where $\boldsymbol{Q}$ is a square matrix whose $i$-th column is the eigenvector $\boldsymbol{\nu}_{\boldsymbol{i}}$ and $\boldsymbol{\Lambda}$ is the diagonal matrix whose elements are the corresponding eigenvalues $\lambda_{i}$ (i.e., $\Lambda_{i i}=\lambda_{i}$, and $\Lambda_{i j}=0$ for $i \neq j$ ). A useful approximation can be obtained if $\lambda_{d+1}$ through $\lambda_{p}$ are (relatively) small

$$
\boldsymbol{\Sigma} \approx \boldsymbol{Q} \boldsymbol{\Lambda}_{d} \boldsymbol{Q}^{-1}
$$

where the matrix $\boldsymbol{\Lambda}_{d}$ is the truncation of $\boldsymbol{\Lambda}$ keeping only the first $d$ largest eigenvalues. More precisely, the amount of the variation explained by each component is given by

$$
\bar{\lambda}_{i}=\frac{\lambda_{i}}{\sum_{j} \lambda_{j}}
$$

Then, the first $d$ chosen principal components form an orthonormal basis. Hence any strain vector $\boldsymbol{X}$ can be projected onto this subspace as $\boldsymbol{Z}_{d}$

$$
\boldsymbol{Z}_{d}=\boldsymbol{P}_{d} \boldsymbol{X}
$$

with the projection operator $\boldsymbol{P}_{d}$

$$
\boldsymbol{P}_{d}=\boldsymbol{Q} \boldsymbol{I}_{d} \boldsymbol{Q}^{\top}
$$

and the truncated identity $\boldsymbol{I}_{d}$.

As such, a dataset is formed from 8740 strain measurements in Voigt notation. The corresponding eigenvectors and eigenvalues are shown in tables 1 and 2. Given that the first three eigenvalues represent a reasonable amount of the variance (i.e., almost 90\%), only the three first principal components are chosen for the new basis $d=3$. 
Table 1: Principal components for the relative strains dataset, the retained eigenvectors are shown in boldface

\begin{tabular}{lrrrrrr}
\hline$\nu$ & $\epsilon_{x x}$ & $\epsilon_{y y}$ & $\epsilon_{z z}$ & $\epsilon_{y z}$ & $\epsilon_{x z}$ & $\epsilon_{x y}$ \\
\hline $\mathbf{1}$ & $\mathbf{- 6 . 3} \%$ & $\mathbf{- 6 . 5} \%$ & $\mathbf{0 . 4} \%$ & $\mathbf{5 . 6} \%$ & $\mathbf{- 8 . 0} \%$ & $\mathbf{- 9 9 . 1} \%$ \\
$\mathbf{2}$ & $\mathbf{9 2 . 7} \%$ & $\mathbf{- 2 7 . 3} \%$ & $\mathbf{0 . 4} \%$ & $\mathbf{2 5 . 3} \%$ & $\mathbf{- 4 . 5} \%$ & $\mathbf{- 2 . 3} \%$ \\
$\mathbf{3}$ & $\mathbf{- 3 2 . 7} \%$ & $\mathbf{- 2 8 . 4} \%$ & $\mathbf{7 . 3} \%$ & $\mathbf{8 9 . 2} \%$ & $\mathbf{- 5 . 5} \%$ & $\mathbf{9 . 5} \%$ \\
4 & $-17.0 \%$ & $-91.6 \%$ & $1.9 \%$ & $-36.0 \%$ & $1.5 \%$ & $5.0 \%$ \\
5 & $3.2 \%$ & $1.8 \%$ & $76.8 \%$ & $-0.1 \%$ & $63.8 \%$ & $-5.2 \%$ \\
6 & $-0.1 \%$ & $4.0 \%$ & $63.6 \%$ & $-9.3 \%$ & $-76.3 \%$ & $5.6 \%$ \\
\hline
\end{tabular}

Table 2: Amount of individual and cumulative variance explained by each principal components, the eigenvalues associated to the retained eigenvectors are shown in boldface

\begin{tabular}{rrrr}
\hline$\nu$ & $\lambda$ & $\bar{\lambda}$ & $\sum \bar{\lambda}$ \\
\hline $\mathbf{1}$ & $\mathbf{0 . 0 2 5 9}$ & $\mathbf{5 8 . 5} \%$ & $\mathbf{5 8 . 5} \%$ \\
$\mathbf{2}$ & $\mathbf{0 . 0 0 8 8}$ & $\mathbf{1 9 . 9} \%$ & $\mathbf{7 8 . 4} \%$ \\
$\mathbf{3}$ & $\mathbf{0 . 0 0 4 6}$ & $\mathbf{1 0 . 5} \%$ & $\mathbf{8 8 . 9} \%$ \\
4 & 0.0031 & $7.1 \%$ & $96.0 \%$ \\
5 & 0.0010 & $2.2 \%$ & $98.2 \%$ \\
6 & 0.0008 & $1.8 \%$ & $100.0 \%$ \\
\hline
\end{tabular}

This provides the projected dataset $\boldsymbol{Z}_{3}$, as shown in figure 9 , with the principal components being mostly aligned with $\epsilon_{x y}(-99.1 \%), \epsilon_{x x}(92.7 \%)$ and $\epsilon_{y z}$ $(89.2 \%)$, respectively. One can observe three distinct groups. The first two groups (along $\nu_{1}$ ) are symmetrically opposed, they represent shear strain in the 
$(x-y)$ plane with opposing magnitudes. On the contrary, the third group (aligned with $\nu_{2}$ ) represents the compression (normal strain) along the $x$ axis. Also, the first two groups are symmetrically opposed to the third group (along $\nu_{3}$ ). Indeed they represent shear strain in the $(y-z)$ plane with opposing magnitudes.

Regarding the principal components, it is noteworthy that the influence of $\epsilon_{x z}$ and $\epsilon_{z z}$ is very low. As expected from the observed layered kinematics, they represent almost no activity in the transversal orientation of the composite (between layers).
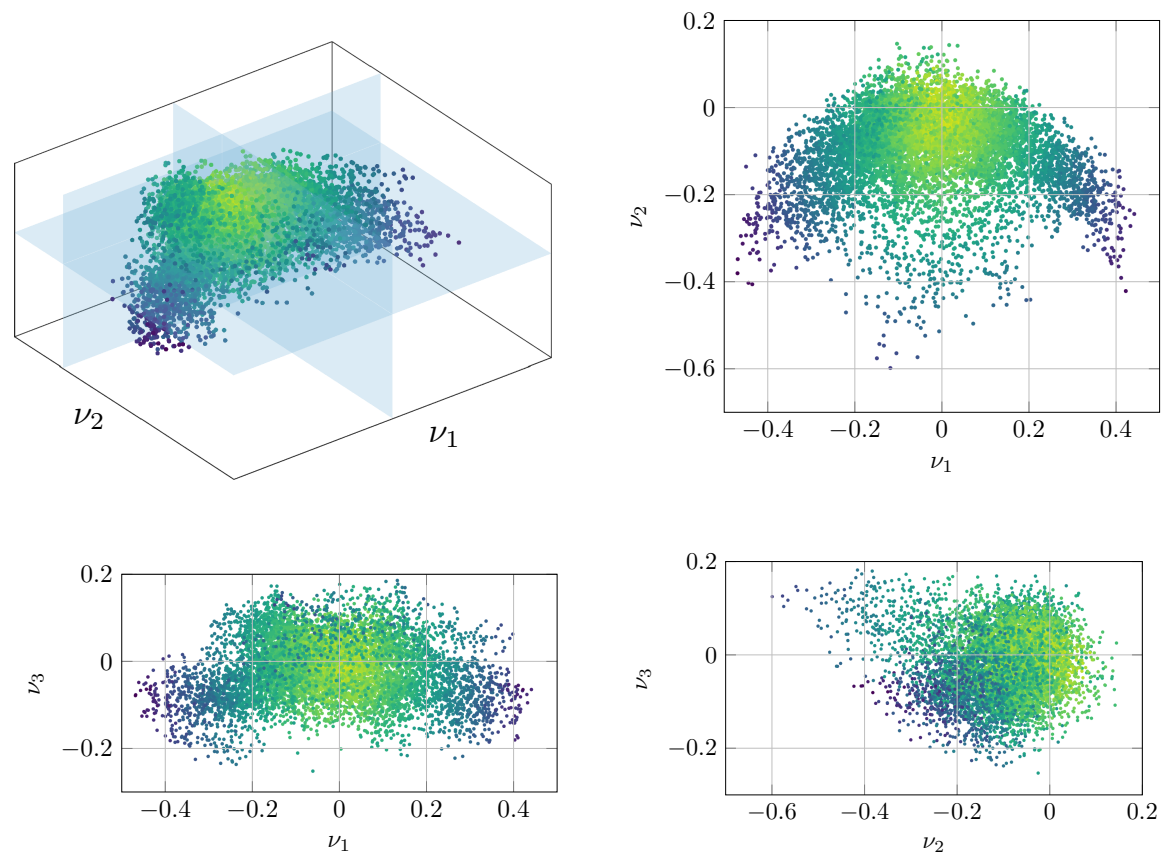

Figure 9: Embedding of the dataset onto a two-dimensional space using PCA

It should be noted that these described "groups" belong a continuous evolution of a phenomenon. That is, if $\epsilon$ is identified to belong to a group, then $\alpha * \epsilon$ for any $0<\alpha<1$ is likely to happen and should belong to the same category. As such, the identification of (typology of) strain patterns should be independent of its magnitude. Naturally, this calls for normalizing each strain 
value by its norm (i.e., the norm associated to the full tensor), an histogram of these magnitudes is shown in figure 10. Now the problem is reduced to identifying the groups of patterns at the surface of the (6D) unit sphere. Furthermore, given that points with low activity (i.e., norm smaller than $\epsilon_{o}$ ) do not provide reliable information about the sought orientations, they are removed from the analysis. This subset of normalized points projected onto the basis defined by $\boldsymbol{P}_{3}$ is shown in figure 12. For visualization purposes a (zero centered) ellipsoid is fitted to the data using Linear Least Squares, this represents the unit sphere projection.

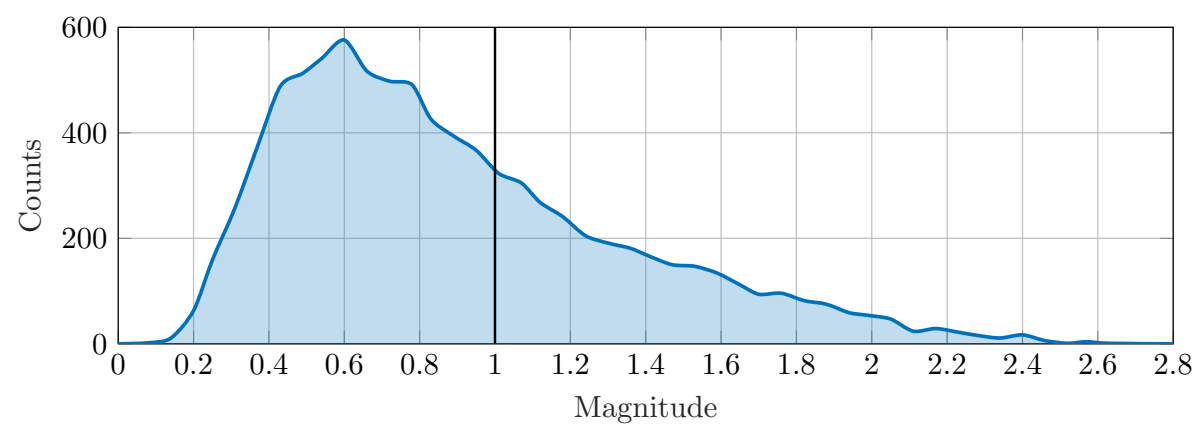

Figure 10: Histogram of the strain norms (normalized with respect to $\epsilon_{o}$ )

The identification of these groups will be performed via $k$-means $[55,56]$. This clustering operation defines groups (i.e., clusters) so that data points belonging to the same group are more similar (according to some metric) to each other than those in other groups. It is an iterative procedure that minimizes the (euclidean) distance between data points and the centroid of the identified clusters, which are then updated. The initialization of the clusters is done via the $k$-means ++ algorithm [57], which uses a simple heuristic to find centroid seeds: them being initially spread out is desirable. This initialization procedure is known to improve the convergence and quality of the final solution.

As previously identified, the selected number of strain patterns is three. As such, using $k$-means on the chosen subset of normalized data points, the cluster centroids are identified. The table 3 lists them using the basis defined by $\boldsymbol{P}_{3}$ (as 
they were computed). The clustered points and identified centroids are shown in figures 11 and 12.

Additionally, they can be listed in the original space (using Voigt notation) or in their invariant forms, as shown in tables 4 and 5 , respectively. They further highlight the predominant planar nature of the relative strains (i.e., $\epsilon_{3} \approx 0$ ), as illustrated by the glyphs.

Table 3: Centroids found using $k$-means

\begin{tabular}{rrrr}
\hline$K$ & $\nu_{1}$ & $\nu_{2}$ & $\nu_{3}$ \\
\hline 1 & $-58.0 \%$ & $-32.4 \%$ & $-9.3 \%$ \\
2 & $58.5 \%$ & $-34.7 \%$ & $-13.1 \%$ \\
3 & $1.4 \%$ & $-85.5 \%$ & $17.5 \%$ \\
\hline
\end{tabular}

Table 4: Centroids projected into the original space (Voigt notation)

\begin{tabular}{rrrrrrr}
\hline$K$ & $\epsilon_{x x}$ & $\epsilon_{y y}$ & $\epsilon_{z z}$ & $\epsilon_{y z}$ & $\epsilon_{x z}$ & $\epsilon_{x y}$ \\
\hline 1 & $-23.3 \%$ & $15.3 \%$ & $-1.0 \%$ & $-19.7 \%$ & $6.6 \%$ & $57.4 \%$ \\
2 & $-31.6 \%$ & $9.4 \%$ & $-0.9 \%$ & $-17.2 \%$ & $-2.4 \%$ & $-58.5 \%$ \\
3 & $-85.1 \%$ & $18.3 \%$ & $1.0 \%$ & $-5.9 \%$ & $2.8 \%$ & $2.2 \%$ \\
\hline
\end{tabular}

Table 5: Centroids expressed using their invariant forms

\begin{tabular}{rrrrrrr}
\hline$K$ & $\operatorname{tr}(\boldsymbol{\epsilon})$ & $\boldsymbol{\epsilon}_{e q}$ & $\epsilon_{1}$ & $\epsilon_{2}$ & $\epsilon_{3}$ & glyph \\
\hline 1 & $-9.1 \%$ & $22.4 \%$ & $-68.8 \%$ & $59.1 \%$ & $0.7 \%$ & 0 \\
2 & $-23.0 \%$ & $24.6 \%$ & $-75.0 \%$ & $53.8 \%$ & $-1.9 \%$ & \\
3 & $-65.8 \%$ & $63.9 \%$ & $-85.2 \%$ & $20.1 \%$ & $-0.7 \%$ & \\
\hline
\end{tabular}



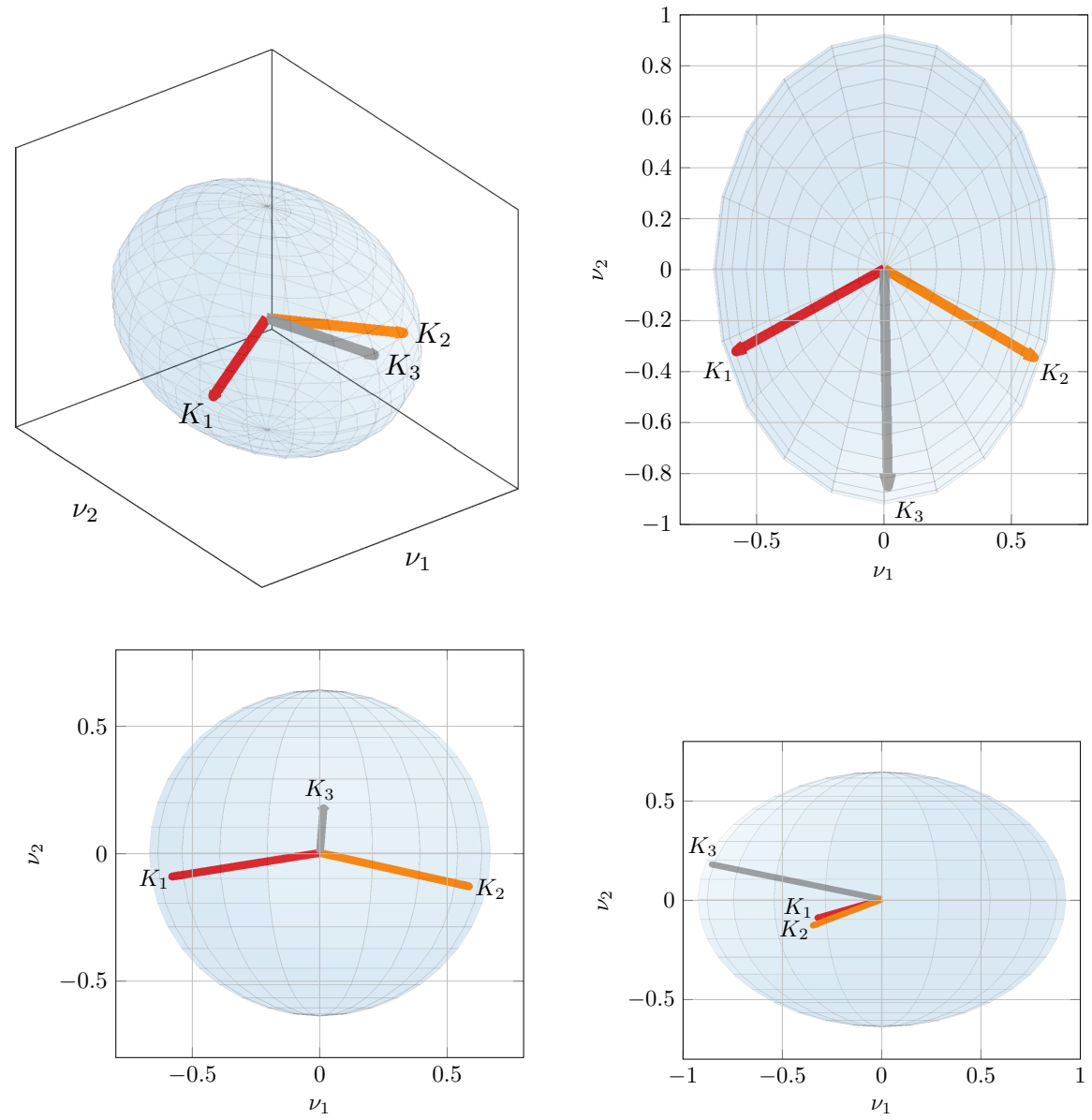

Figure 11: Centroids found using $k$-means, the position of the centroids is indicated by the arrows

Finally, a volume representation of the different groups is shown in figure 13. The original data points (whose spatial coordinates are known) are shown for each cluster. As expected, the clustering analysis identifies the previously described strain patterns. It helps "summarizing" the relatively complex phenomena into quantifiable descriptors. They build upon the qualitative analysis first presented. 

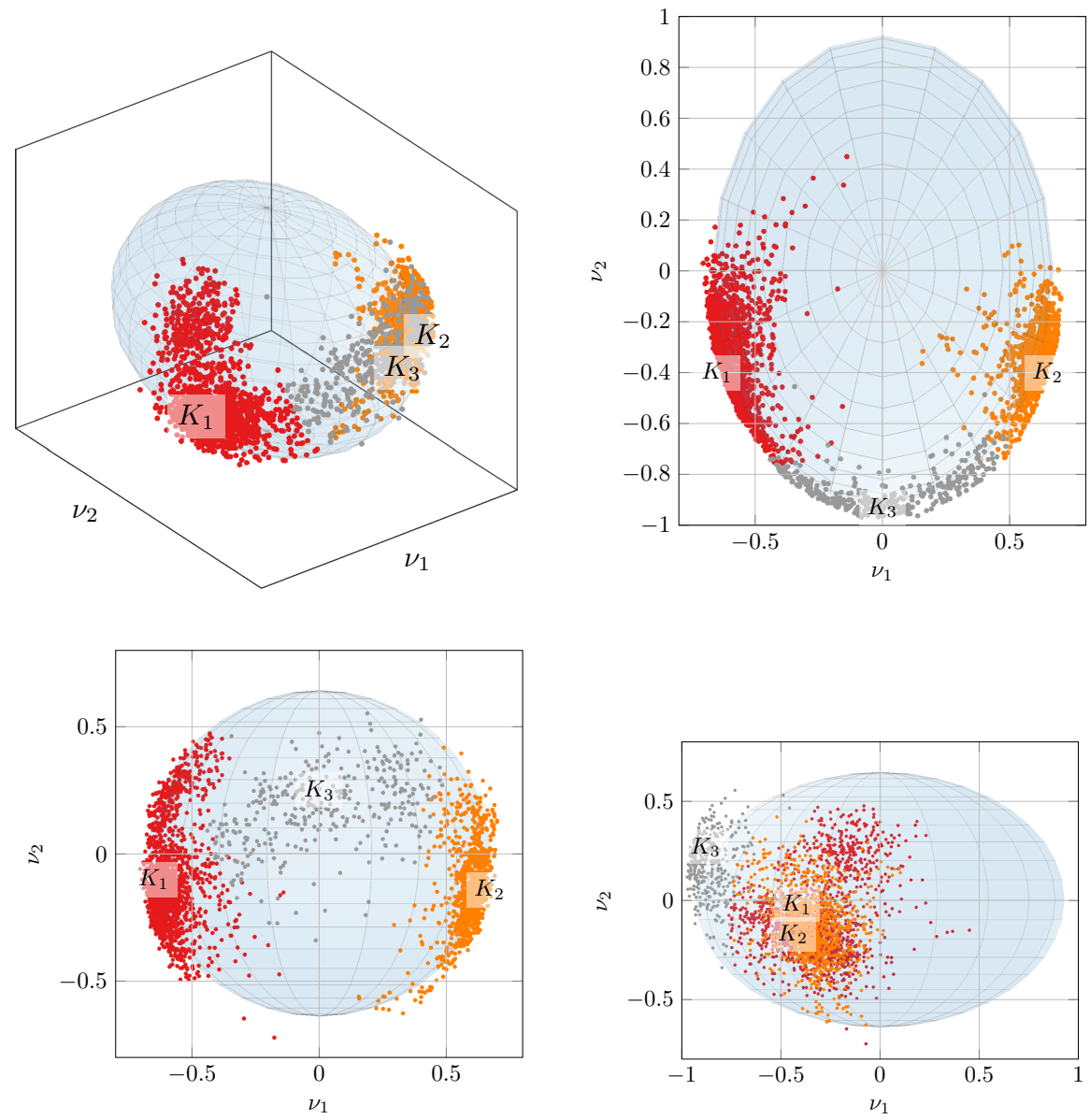

Figure 12: Clusters formed using a subset of points embedded in the space defined using PCA 


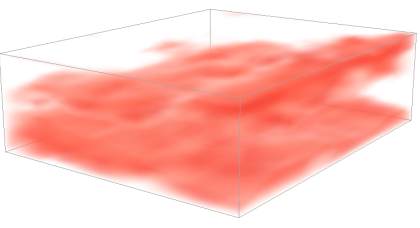

(a) Cluster for $K_{1}$

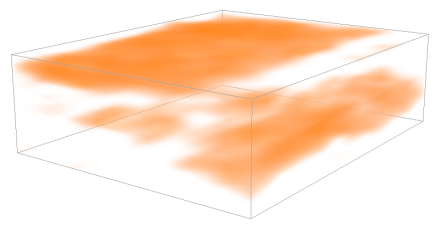

(b) Cluster for $K_{2}$

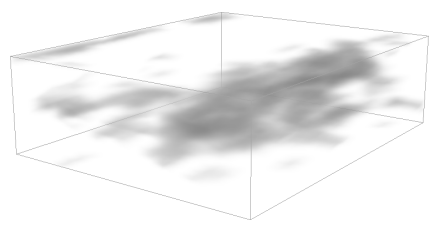

(c) Cluster for $K_{3}$

Figure 13: Volume representation of the identified clusters 


\subsection{Combined analysis of results}

The previously identified strain patterns for the pair S3-S4 amount to a reduced vocabulary of deformations. They represent the complex deformations induced by the manufacturing process. As is, it is very limited, as it is sampled from a reduced population (i.e., only one sample pair). Moreover, incorporating more sample pairs into this analysis would provide a common "language" for describing these deformations. Then, once a complete basis has been defined, it can be used for analyses on new unseen samples. As such it helps expressing a multitude of results under a common formalism, thus easing the comparison. Likewise, this basis could replace the previously used FE decomposition, and be employed for identification purposes (e.g., Integrated-DIC [58]).

For illustration purpose, the formed basis (from the pair S3-S4) will be employed for analyzing the results obtained from pair S1-S2. A database of relative strains, projecting them using $\boldsymbol{P}_{3}$ - performing the normalization procedure, and assigning the points to the cluster whose centroid is closer - is constructed. The new points embedded in the reduced space are shown in figure 14. Their normalized counterparts are shown in figure 15 alongside with the clusters they belong to. Similarly, a volume representation of the clusters is provided in figure 16. 

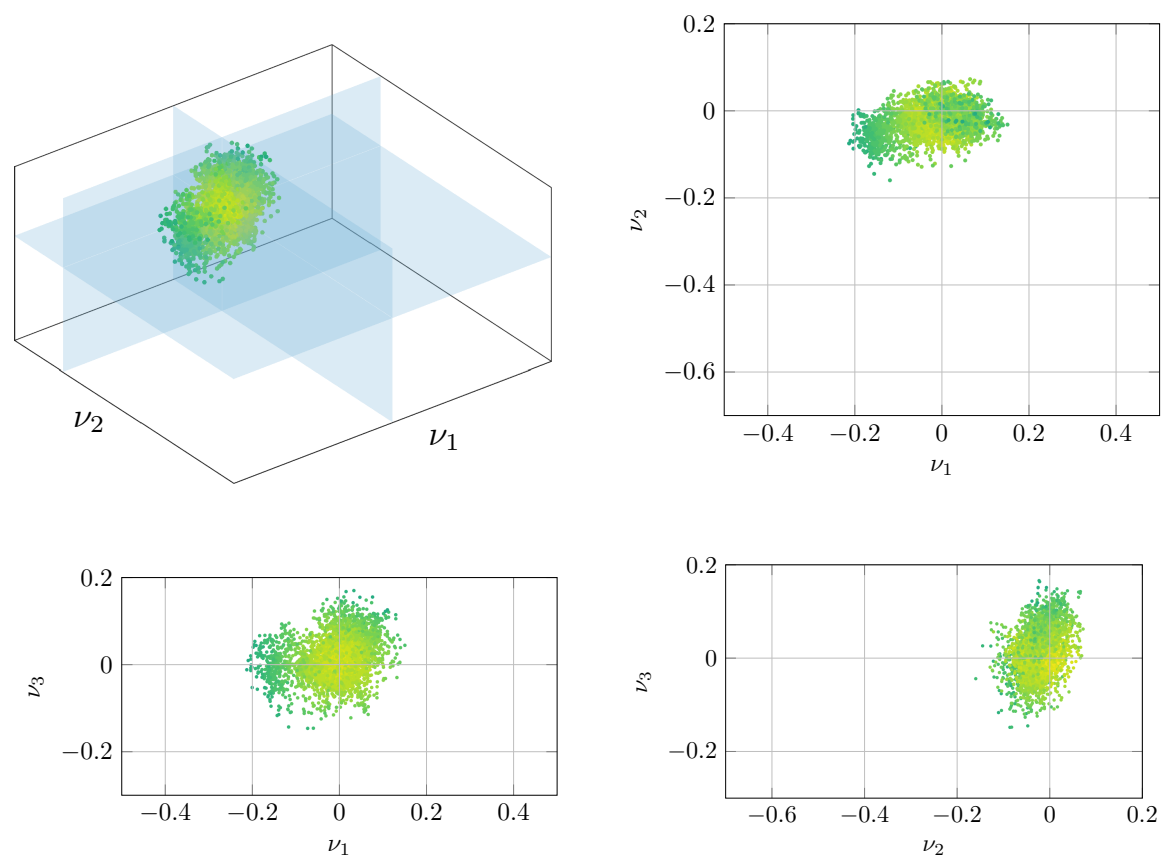

Figure 14: Embedding of the dataset onto a two-dimensional space using PCA 

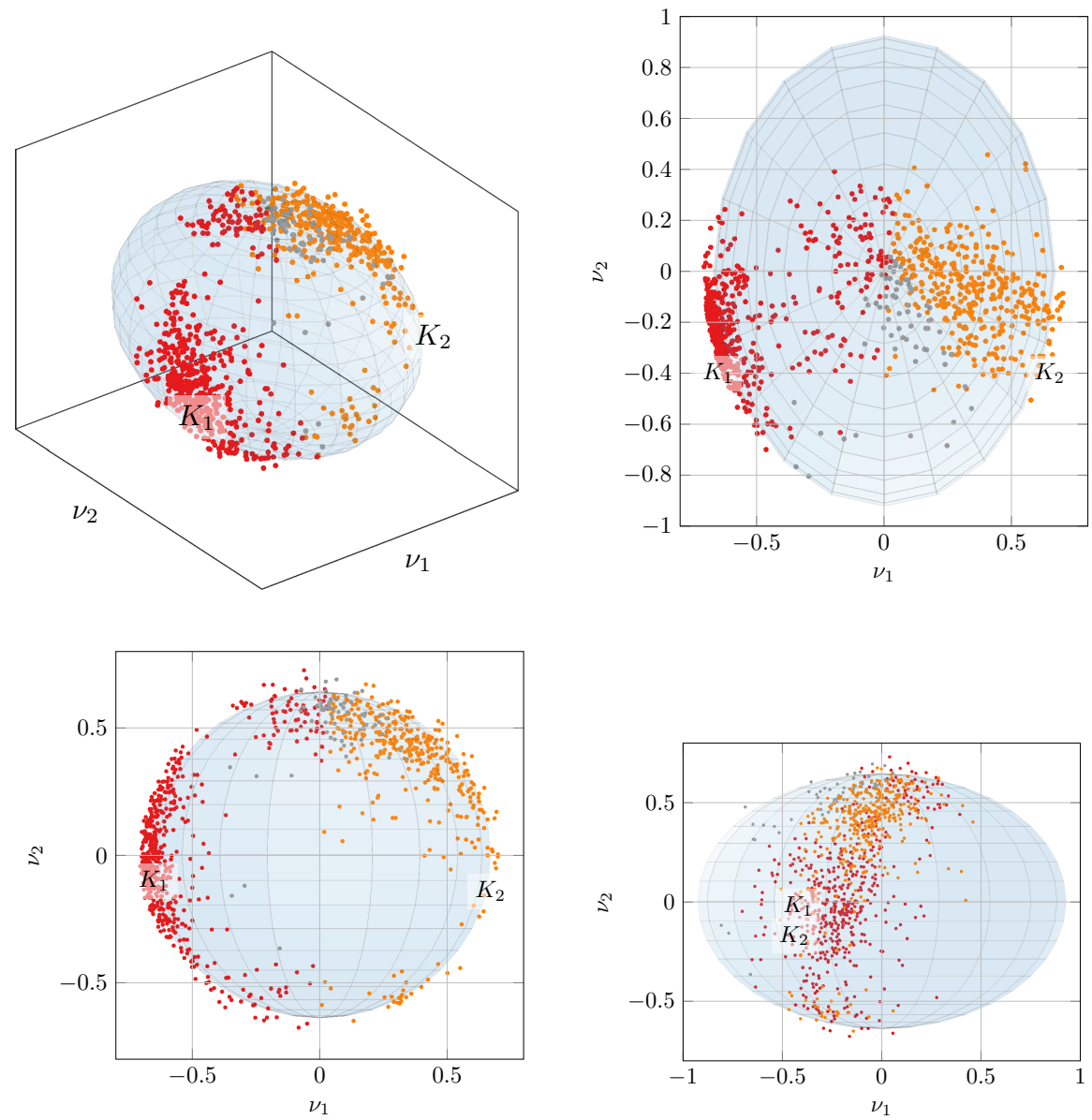

Figure 15: Clusters formed using a subset of points embedded in the space defined using PCA 


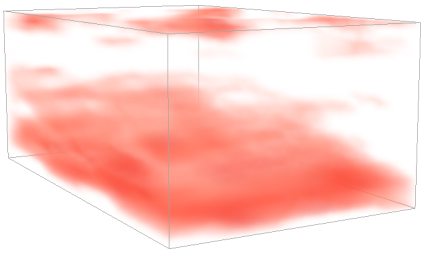

(a) Cluster for $K_{1}$

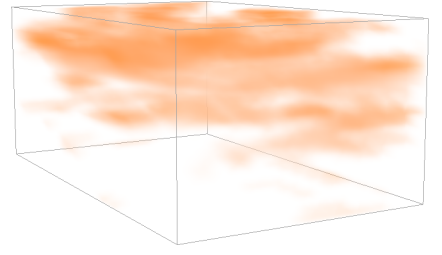

(b) Cluster for $K_{2}$

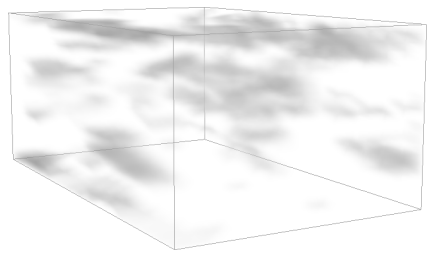

(c) Cluster for $K_{3}$

Figure 16: Volume representation of the identified clusters 


\section{Conclusions}

A novel approach based on DVC has been presented for measuring the yarn deformations induced by the complete manufacturing process of woven composites.

Four woven composite samples observed under similar conditions (tomographic acquisition parameters) are analyzed. From these relative studies, some interesting patterns are inferred. They inform on "sliding" between the layers of the woven composite that occur during the manufacturing process. This layered kinematics is motivated by the fact that each layer suffers almost to none in-plane deformation (along warp or weft orientation), thus leaving mostly rigid translation. Such measured phenomena does agree with some previously observed (qualitative) deformations. Moreover, the results were presented using a diverse set of visualization techniques, each aiming at better displaying a certain feature. Indeed, pinpointing the "best" representation of the results (for all tasks) is a difficult assignment. However, identifying the one that best fits a particular need (for a given task) should prove apparent.

These relative strains are then analyzed using unsupervised learning techniques so as to create a reduced vocabulary of strains patterns. For the construction of this "dictionary" of patterns, the learning was performed on only a pair of samples and the testing was performed on the remaining pair of samples. It should be noted that the current reduced number of studied samples (and reduced number of patterns) is not sufficient to perform a thorough statistical analysis of the phenomena. For such reason, further experimental investigations are planned. An additional advantage of employing such vocabulary, is that it can serve as a basis for the kinematic decomposition and thus be employed for identification purposes [58].

Just as DIC (and Stereo-DIC) were paramount for the validation of textile forming models, this technique has the potential to do so for 3D. Since the proposed approach allows the quantitative analysis of the entire sample (not only the surface), it provides a complete description of the deformations induced 
by the manufacturing process or external factors. Indeed, this technique can be re-purposed if the deformations induced by each step of the manufacturing process is desired. This would necessitate tomographic scans before and after a particular process (e.g., weaving, forming, injection).

Similarly, the characterization of the so-called realistic "as-woven" configurations could be enabled by this method. This would imply a rigorous statistical analysis on multiple formed samples (prior to injection). Naturally, this technique has the potential for analyzing the deformations induced by complex shapes.

Furthermore, the proposed approach could be employed along some textile forming models. In particular, with those employing a reduced number of "simple" parameters (e.g., not employing textile pre-processors [33]). Then, the impact of these parameters on the final as-woven configuration could be expressed using the measured strain patterns. As such, the validation of the models (and the parameters) could be performed automatically.

Interestingly, this method can also be employed for a deeper comprehension of the whole manufacturing process itself. As such, it opens the possibility for a sensitivity analysis on the many parameters involved in the manufacturing process. In fact, the evolution of any given parameter could be instead expressed as an evolution on the material at any stage.

It is important to note that the presented application of DVC helps overcome the many challenges related to the analysis of volume data obtained via $\mathrm{X}$ ray micro-CT. Not only the calculations are performed without (tedious and long) manual operation, but they also provide full-field measurements instead of qualitative observations.

Finally, an extension of the technique consists in endowing it with "unwrapping" [59] capabilities so as to relate the observed phenomena with the underlying weaving pattern. As such, the impact of the different aspects of the manufacturing process can be understood with respect to the textile architecture. This approach is currently being explored. 


\section{Acknowledgements}

This work was supported by the PRC MECACOMP, project co-founded by DGAC and Safran group, involving Safran group companies, ONERA, CNRS and other academic partners. AMQ acknowledges the support of a $\mathrm{PhD}$ grant from ANRT and Safran Tech.

[1] A. Mendoza, J. Schneider, E. Parra, E. Obert, S. Roux, Differentiating 3D textile composites: A novel field of application for Digital Volume Correlation, Composite Structures 208 (2018) 735-743, ISSN 02638223, 10.1016/j.compstruct.2018.10.008.

[2] F. Desplentere, S. V. Lomov, D. L. Woerdeman, I. Verpoest, M. Wevers, A. Bogdanovich, Micro-CT characterization of variability in 3D textile architecture, Composites Science and Technology 65 (13) (2005) 1920-1930, ISSN 02663538, 10.1016/j.compscitech.2005.04.008.

[3] T. Gereke, O. Döbrich, M. Hübner, C. Cherif, Experimental and computational composite textile reinforcement forming: A review, Composites Part A: Applied Science and Manufacturing 46 (1) (2013) 1-10, ISSN 1359835X, 10.1016/j.compositesa.2012.10.004.

[4] P. Boisse, J. Colmars, N. Hamila, N. Naouar, Q. Steer, Bending and wrinkling of composite fiber preforms and prepregs. A review and new developments in the draping simulations, Composites Part B: Engineering 141 (2018) 234-249, ISSN 13598368, 10.1016/j.compositesb.2017.12.061.

[5] M. Sutton, W. Wolters, W. Peters, W. Ranson, S. McNeill, Determination of displacements using an improved digital correlation method, Image and Vision Computing 1 (3) (1983) 133-139, ISSN 02628856, 10.1016/0262-8856(83)90064-1.

[6] T. C. Chu, W. F. Ranson, M. A. Sutton, Applications of digital-imagecorrelation techniques to experimental mechanics, Experimental Mechanics 25 (3) (1985) 232-244, ISSN 17412765, 10.1007/BF02325092. 
[7] H. A. Bruck, S. R. McNeill, M. A. Sutton, W. H. Peters, Digital image correlation using Newton-Raphson method of partial differential correction, Experimental mechanics 29 (3) (1989) 261-267.

[8] M. A. Sutton, J. H. Yan, V. Tiwari, H. W. Schreier, J. J. Orteu, The effect of out-of-plane motion on 2D and 3D digital image correlation measurements, Optics and Lasers in Engineering 46 (10) (2008) 746-757, 10.1016/j.optlaseng.2008.05.005.

[9] A. C. Long, C. D. Rudd, M. Blagdon, P. Smith, Characterizing the processing and performance of aligned reinforcements during preform manufacture, Composites Part A: Applied Science and Manufacturing 27 (4 PART A) (1996) 247-253, ISSN 1359835X, 10.1016/1359-835X(95)00051-3.

[10] H. Lin, J. Wang, A. C. Long, M. J. Clifford, P. Harrison, Predictive modelling for optimization of textile composite forming, Composites Science and Technology 67 (15-16) (2007) 3242-3252, ISSN 02663538, 10.1016/j.compscitech.2007.03.040.

[11] S. V. Lomov, D. S. Ivanov, I. Verpoest, M. Zako, T. Kurashiki, H. Nakai, J. Molimard, A. Vautrin, Full-field strain measurements for validation of meso-FE analysis of textile composites, Composites Part A: Applied Science and Manufacturing 39 (8) (2008) 1218-1231, ISSN 1359835X, 10.1016/j.compositesa.2007.09.011.

[12] X. K. Li, S. L. Bai, Sheet forming of the multi-layered biaxial weft knitted fabric reinforcement. Part I: On hemispherical surfaces, Composites Part A: Applied Science and Manufacturing 40 (6-7) (2009) 766-777, ISSN 1359835X, 10.1016/j.compositesa.2009.03.007.

[13] K. Vanclooster, S. V. Lomov, I. Verpoest, Experimental validation of forming simulations of fabric reinforced polymers using an unsymmetrical mould configuration, Composites Part A: Applied Science and Manufacturing 40 (4) (2009) 530-539, ISSN 1359835X, 10.1016/j.compositesa.2009.02.005. 
[14] M. A. Khan, T. Mabrouki, E. Vidal-Sallé, P. Boisse, Numerical and experimental analyses of woven composite reinforcement forming using a hypoelastic behaviour. Application to the double dome benchmark, Journal of Materials Processing Technology 210 (2) (2010) 378-388, ISSN 09240136, 10.1016/j.jmatprotec.2009.09.027.

[15] S. Allaoui, P. Boisse, S. Chatel, N. Hamila, G. Hivet, D. Soulat, E. VidalSalle, Experimental and numerical analyses of textile reinforcement forming of a tetrahedral shape, Composites Part A: Applied Science and Manufacturing 42 (6) (2011) 612-622, ISSN 1359835X, 10.1016/j.compositesa.2011. 02.001 .

[16] S. Gatouillat, A. Bareggi, E. Vidal-Sallé, P. Boisse, Meso modelling for composite preform shaping - Simulation of the loss of cohesion of the woven fibre network, Composites Part A: Applied Science and Manufacturing 54 (2013) 135-144, ISSN 1359835X, 10.1016/j.compositesa.2013.07.010.

[17] B. K. Bay, T. S. Smith, D. P. Fyhrie, M. Saad, Digital volume correlation: Three-dimensional strain mapping using X-ray tomography, Experimental Mechanics 39 (3) (1999) 217-226, ISSN 0014-4851, 10.1007/BF02323555.

[18] B. K. Bay, Methods and applications of digital volume correlation, The Journal of Strain Analysis for Engineering Design 43 (8) (2008) 745-760, ISSN 0309-3247, 10.1243/03093247JSA436.

[19] Y. Wang, X. Sun, Digital-element simulation of textile processes, Composites Science and Technology 61 (2) (2001) 311-319, ISSN 02663538, 10.1016/S0266-3538(00)00223-2.

[20] A. Drach, B. Drach, I. Tsukrov, Processing of fiber architecture data for finite element modeling of 3D woven composites, Advances in Engineering Software 72 (2014) 18-27, ISSN 18735339, 10.1016/j.advengsoft.2013.06. 006. 
[21] B. Chen, T. W. Chou, Compaction of woven-fabric preforms in liquid composite molding processes: Single-layer deformation, Composites Science and Technology 59 (10) (1999) 1519-1526, ISSN 02663538, 10.1016/ S0266-3538(99)00002-0.

[22] P. Potluri, I. Parlak, R. Ramgulam, T. V. Sagar, Analysis of tow deformations in textile preforms subjected to forming forces, Composites Science and Technology 66 (2) (2006) 297-305, ISSN 02663538, 10.1016/j. compscitech.2005.04.039.

[23] Z. R. Chen, L. Ye, A micromechanical compaction model for woven fabric preforms. Part II: Multilayer, Composites Science and Technology 66 (16) (2006) 3263-3272, ISSN 02663538, 10.1016/j.compscitech.2005.07.010.

[24] S. V. Lomov, D. S. Ivanov, I. Verpoest, M. Zako, T. Kurashiki, H. Nakai, S. Hirosawa, Meso-FE modelling of textile composites: Road map, data flow and algorithms, Composites Science and Technology 67 (9) (2007) 1870-1891, ISSN 02663538, 10.1016/j.compscitech.2006.10.017.

[25] F. Stig, S. Hallström, Spatial modelling of 3D-woven textiles, Composite Structures 94 (5) (2012) 1495-1502, ISSN 02638223, 10.1016/j.compstruct. 2011.12.003.

[26] F. Stig, S. Hallström, A modelling framework for composites containing 3D reinforcement, Composite Structures 94 (9) (2012) 2895-2901, ISSN 02638223, 10.1016/j.compstruct.2012.03.009.

[27] G. Zhou, X. Sun, Y. Wang, Multi-chain digital element analysis in textile mechanics, Composites Science and Technology 64 (2) (2004) 239-244, ISSN 02663538, 10.1016/S0266-3538(03)00258-6.

[28] Y. Miao, E. Zhou, Y. Wang, B. A. Cheeseman, Mechanics of textile composites: Micro-geometry, Composites Science and Technology 68 (7-8) (2008) 1671-1678, ISSN 02663538, 10.1016/j.compscitech.2008.02.018. 
[29] E. Zhou, D. Mollenhauer, E. Iarve, A realistic 3-D textile geometric model, in: Seventeenth international conference on composite materials ICCM-17, 100-110, 2009.

[30] Y. Mahadik, S. R. Hallett, Finite element modelling of tow geometry in 3D woven fabrics, Composites Part A: Applied Science and Manufacturing 41 (9) (2010) 1192-1200, ISSN 1359835X, 10.1016/j.compositesa.2010.05. 001.

[31] D. Durville, Numerical simulation of entangled materials mechanical properties, Journal of Materials Science 40 (22) (2005) 5941-5948, ISSN 00222461, 10.1007/s10853-005-5061-2.

[32] S. D. Green, A. C. Long, B. S. El Said, S. R. Hallett, Numerical modelling of 3D woven preform deformations, Composite Structures 108 (1) (2014) 747-756, ISSN 02638223, 10.1016/j.compstruct.2013.10.015.

[33] D. Durville, I. Baydoun, H. Moustacas, G. Périé, Y. Wielhorski, Determining the initial configuration and characterizing the mechanical properties of 3D angle-interlock fabrics using finite element simulation, International Journal of Solids and Structures ISSN 00207683, 10.1016/j.ijsolstr.2017.06. 026.

[34] N. Naouar, E. Vidal-Salle, E. Maire, J. Schneider, P. Boisse, Meso F.E. simulation of composite reinforcement deformation based on X-ray computed tomography, Materiaux \& Techniques 104 (4) (2014) 165-176, ISSN 0032-6895, 10.1051/mattech/2016029.

[35] N. Naouar, E. Vidal-Salle, J. Schneider, E. Maire, P. Boisse, 3D composite reinforcement meso F.E. analyses based on X-ray computed tomography, Composite Structures 132 (2015) 1094-1104, 10.1016/j.compstruct.2015. 07.005 .

[36] A. Mendoza, J. Neggers, F. Hild, S. Roux, Complete Mechanical Regularization Applied To Digital Image and Volume Correlation, 2018. 
[37] D. Claire, F. Hild, S. Roux, A finite element formulation to identify damage fields: the equilibrium gap method, International Journal for Numerical Methods in Engineering 61 (2) (2004) 189-208.

[38] S. Roux, F. Hild, H. Leclerc, Mechanical assistance to DIC, Procedia IUTAM 4 (2012) 159-168, ISSN 22109838, 10.1016/j.piutam.2012.05.018.

[39] Z. Tomicevic, F. Hild, S. Roux, Mechanics-Aided Digital Image Correlation, The Journal of Strain Analysis for Engineering Design 48 (5) (2013) 330343.

[40] B. Xin, J. Hu, G. Baciu, X. Yu, Development of weave code technology for textile products, Fibres \& Textiles in Eastern Europe (2011) 33-35.

[41] O. C. Zienkiewicz, The finite element method, vol. 3, McGraw-hill London, 1977.

[42] H. Leclerc, J. N. Périé, S. Roux, F. Hild, Voxel-Scale Digital Volume Correlation, Experimental Mechanics 51 (4) (2011) 479-490, ISSN 00144851, 10.1007/s11340-010-9407-6.

[43] E. Süli, D. F. Mayers, An introduction to numerical analysis, Cambridge university press, 2003.

[44] A. Mendoza, S. Roux, J. Schneider, E. Parra, E. Obert, S. Roux, Differentiating 3D textile composites: A novel field of application for Digital Volume Correlation, 7th Conference on Industrial Computed Tomography 208 (iCT) (2017) 1-6, ISSN 02638223, 10.1016/j.compstruct.2018.10.008.

[45] H. Hencky, Ober die Form des ElastizitUtsgesetzes bei ideal elastischen Stoffen, Journal of Rheology 2 (1928) 169-176.

[46] S. Onaka, Appropriateness of the Hencky Equivalent Strain as the Quantity to Represent the Degree of Severe Plastic Deformation, MATERIALS TRANSACTIONS 53 (8) (2012) 1547-1548, 10.2320/matertrans. M2012077. 
[47] W. Voigt, Lehrbuch der Kristallphysik, Vieweg+Teubner Verlag, Wiesbaden, ISBN 978-3-663-15316-0, 10.1007/978-3-663-15884-4, 1966.

[48] S. C. Shrivastava, J. J. Jonas, G. Canova, Equivalent strain in large deformation torsion testing Theoretical and practical considerations, J. Mech. Phys. Solids 30 (2) (1982) 75-90.

[49] S. Shrivastava, C. Ghosh, J. J. Jonas, A comparison of the von Mises and Hencky equivalent strains for use in simple shear experiments, Philosophical Magazine 92 (18) (2012) 2264-2271, ISSN 14786435, 10.1080/14786435. 2012.671551.

[50] P. J. Basser, C. Pierpaoli, Microstructural and physiological features elucidated by quantitative diffusion tensor $\{\mathrm{MRI}\}$, J. Magnetic Resonance, B 111 (1996) 209-219.

[51] G. Kindlmann, Superquadric Tensor Glyphs, Joint Eurographics - IEEE TCVG Symposium on Visualization (2004) 1-8ISSN 1727-5296, 10.2312/ VisSym/VisSym04/147-154.

[52] K. Pearson, On lines and planes of closest fit to systems of points in space, Philosophical Magazine Series 62 (11) (1901) 559-572, ISSN 1941-5982, 10.1080/14786440109462720.

[53] H. Hotelling, Analysis of a complex of statistical variables into principal components, Journal of Educational Psychology 24 (6) (1933) 417-441, ISSN 00220663, 10.1037/h0071325.

[54] I. Jolliffe, Principal Component Analysis, in: International Encyclopedia of Statistical Science, Springer Berlin Heidelberg, Berlin, Heidelberg, 10941096, 10.1007/978-3-642-04898-2\{_\}455, 2011.

[55] J. MacQueen, Some methods for classification and analysis of multivariate observations, in: Proceedings of the fifth Berkeley symposium on mathematical statistics and probability, 14, Oakland, CA, USA, 281-297, 1967. 
[56] S. P. Lloyd, Least Squares Quantization in PCM, IEEE Transactions on Information Theory 28 (2) (1982) 129-137, ISSN 15579654, 10.1109/TIT. 1982.1056489.

[57] D. Arthur, S. Vassilvitskii, K-Means++: the Advantages of Careful Seeding, Proceedings of the eighteenth annual ACM-SIAM symposium on Discrete algorithms 8 (2007) 1027-1025, ISSN 978-0-898716-24-5, 10.1145/ 1283383.1283494 .

[58] J. Neggers, J. P. M. Hoefnagels, M. G. D. Geers, F. Hild, S. Roux, Timeresolved integrated digital image correlation, International Journal for $\mathrm{Nu}-$ merical Methods in Engineering 103 (3) (2015) 157-182, ISSN 00295981, $10.1002 /$ nme.4882.

[59] A. Mendoza, S. Roux, J. Schneider, E. Parra, E. Obert, Unwrapping Textile Fabric, in: 3rd International Conference on Tomography of Materials and Structures, 1-2, 2017. 